\title{
Front Matter: Volume 9534
}

, "Front Matter: Volume 9534," Proc. SPIE 9534, Twelfth International Conference on Quality Control by Artificial Vision 2015, 953401 (30 April 2015); doi: 10.1117/12.2196823

SPIE Event: The International Conference on Quality Control by Artificial Vision SPIE. 2015, 2015, Le Creusot, France 


\title{
Twelfth International Conference on Quality Control by Artificial Vision
}

\author{
Fabrice Meriaudeau \\ Olivier Aubreton \\ Editors
}

\section{3-5 June 2015 \\ Le Creusot, France}

Organized by

Le2i, CNRS, Université de Bourgogne

Sponsored by

Le Site du Creusot: IUT Le Creusot et Centre Universitaire Condorcet (France)

Université de Bourgogne (France)

CNRS (France)

La Ville du Creusot (France)

La communauté Urbaine Creusot-Montceau (France)

Conseil Régional de Bourgogne (France)

Published by

SPIE 
The papers included in this volume were part of the technical conference cited on the cover and title page. Papers were selected and subject to review by the editors and conference program committee. Some conference presentations may not be available for publication. The papers published in these proceedings reflect the work and thoughts of the authors and are published herein as submitted. The publisher is not responsible for the validity of the information or for any outcomes resulting from reliance thereon.

Please use the following format to cite material from this book:

Author(s), "Title of Paper," in Twelfth International Conference on Quality Control by Artificial Vision, edited by Fabrice Meriaudeau, Olivier Aubreton, Proceedings of SPIE Vol. 9534 (SPIE, Bellingham, WA, 2015) Article CID Number.

ISSN: 0277-786X

ISBN: 9781628416992

\section{Published by}

\section{SPIE}

P.O. Box 10, Bellingham, Washington 98227-0010 USA

Telephone +1 3606763290 (Pacific Time) · Fax +1 3606471445

SPIE.org

Copyright @ 2015, Society of Photo-Optical Instrumentation Engineers.

Copying of material in this book for internal or personal use, or for the internal or personal use of specific clients, beyond the fair use provisions granted by the U.S. Copyright Law is authorized by SPIE subject to payment of copying fees. The Transactional Reporting Service base fee for this volume is $\$ 18.00$ per article (or portion thereof), which should be paid directly to the Copyright Clearance Center (CCC), 222 Rosewood Drive, Danvers, MA 01923. Payment may also be made electronically through CCC Online at copyright.com. Other copying for republication, resale, advertising or promotion, or any form of systematic or multiple reproduction of any material in this book is prohibited except with permission in writing from the publisher. The CCC fee code is 0277-786X/15/\$18.00.

Printed in the United States of America.

Publication of record for individual papers is online in the SPIE Digital Library.

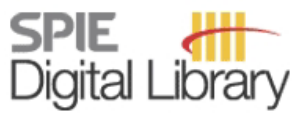

SPIEDigitalLibrary.org

Paper Numbering: Proceedings of SPIE follow an e-First publication model, with papers published first online and then in print. Papers are published as they are submitted and meet publication criteria. A unique citation identifier (CID) number is assigned to each article at the time of the first publication. Utilization of CIDs allows articles to be fully citable as soon as they are published online, and connects the same identifier to all online, print, and electronic versions of the publication. SPIE uses a six-digit CID article numbering system in which:

- The first four digits correspond to the SPIE volume number.

- The last two digits indicate publication order within the volume using a Base 36 numbering

system employing both numerals and letters. These two-number sets start with 00, 01, 02, 03, 04, $05,06,07,08,09,0 A, 0 B \ldots$. OZ, followed by 10-1Z, 20-2Z, etc.

The CID Number appears on each page of the manuscript. The complete citation is used on the first page, and an abbreviated version on subsequent pages. 


\title{
Contents
}

\author{
vii Authors \\ ix Conference Committee \\ xi Introduction
}

SESSION 1 NONCONVENTIONAL IMAGING

953402 Real time polarization imaging of weld pool surface [9534-46]

953403 Semi-automatic classification of cementitious materials using scanning electron microscope images [9534-22]

953404 Standing tree decay detection by using acoustic tomography images [9534-77]

\section{SESSION 2 COLOR AND TEXTURE}

953405 Alternative to colour feature classification using colour contrast ocurrence matrix [9534-11]

953406 Syntactic texture and perception for a new generic visual anomalies classification [9534-40]

953407 A polynomial texture extraction with application in dynamic texture classification [9534-63]

953408 Print spectral reflectance estimation using trichromatic camera [9534-17]

\section{SESSION $3 \quad$ MEDICAL IMAGING AND BIOMEDICAL IMAGING I}

953409 Automatic classification of skin lesions using color mathematical morphology-based texture descriptors [9534-3]

9534 OA A boosting approach for prostate cancer detection using multi-parametric MRI [9534-25]

$9534 \mathrm{OB}$ Joint detection of anatomical points on surface meshes and color images for visual registration of 3D dental models [9534-41]

\section{SESSION 4 MEDICAL IMAGING AND BIOMEDICAL IMAGING II}

9534 OC Breast ultrasound image segmentation: an optimization approach based on super-pixels and high-level descriptors [9534-56]

9534 OD Innovative, non-contact wide field imaging of corneal endothelium [9534-15] 
$9534 \mathrm{OE}$ Characterization of the corneal endothelial mosaic and comparison with simulated tessellations modeled with Gaussian random fields [9534-45]

9534 OF Hardware implementation of fast pupil segmentation using region properties [9534-67]

\section{SESSION $5 \quad$ MACHINE VISION I}

9534 OG Automatic grading of carbon blacks from transmission electron microscopy [9534-64]

$9534 \mathrm{OH} \quad$ Automated visual grading of grain kernels by machine vision [9534-28]

9534 Ol CAD-guided inspection of aeronautical mechanical parts using monocular vision [9534-84]

\section{SESSION 6 MACHINE VISION II}

9534 0J Vision based tunnel inspection using non-rigid registration [9534-92]

9534 OK Local surface orientation analysis based on reflection estimation [9534-30]

$9534 \mathrm{OL} \quad$ A machine vision based approach for timber knots detection [9534-24]

SESSION 7 MULTISPECTRAL AND INFARED IMAGING

9534 OM Cocoa bean quality assessment by using hyperspectral images and fuzzy logic techniques [9534-5]

9534 ON Vector anisotropic filter for multispectral image denoising [9534-14]

953400 Design and calibration of a two-camera (VNIR and SWIR) hyperspectral acquisition system for the characterization of metallic alloys from the recycling industry [9534-37]

9534 OP An efficient method for facial component detection in thermal images [9534-21]

$95340 Q$ Suspicious activity recognition in infrared imagery using Hidden Conditional Random Fields for outdoor perimeter surveillance [9534-76]

\section{SESSION $8 \quad$ METHODS}

9534 OR Noise removal and real-time detail enhancement of high-dynamic-range infrared images with time consistency [9534-71]

9534 OS Improvement of HMM-based action classification by using state transition probability [9534-51]

9534 OT Novel image fusion scheme based on maximum ratio combining for robust multispectral face recognition [9534-89] 
9534 OU A coarse to fine 3D acquisition system [9534-68]

9534 OV A four-lens based plenoptic camera for depth measurements [9534-38]

9534 OW A self-diagnosis under 2D projectivity for local descriptor base template matching [9534-87]

9534 OX A comparative study of multi-sensor data fusion methods for highly accurate assessment of manufactured parts [9534-48]

9534 OY Automated visual inspection of an airplane exterior [9534-35]

$9534 \mathrm{OZ}$ Online fully automated three-dimensional surface reconstruction of unknown objects [9534-62]

SESSION 10 NONDESTRUCTIVE TESTING

953410 Interactive ultrasonic field simulation for nondestructive testing [9534-53]

953411 Tiled fuzzy Hough transform for crack detection [9534-75]

953412 Prediction of fracture profile using digital image correlation [9534-74]

\section{POSTER SESSION}

953413 Robust crack detection strategies for aerial inspection [9534-82]

953414 A stable and unsupervised Fuzzy C-Means for data classification [9534-4]

953415 Ensemble approach for differentiation of malignant melanoma [9534-31]

953416 Intrinsic camera calibration equipped with Scheimpflug optical device [9534-10]

953417 High dynamic range adaptive real-time smart camera: an overview of the HDR-ARTiST project [9534-57]

953418 Hierarchical human action recognition around sleeping using obscured posture information [9534-65]

953419 Calibration of asynchronous smart phone cameras from moving objects [9534-93] 
WORKSHOP ON PHOTOMETRIC STEREO: FROM THEORY TO INDUSTRIAL APPLICATIONS

$95341 \mathrm{~A} \quad$ Real time swallowing measurement system by using photometric stereo [9534-29]

9534 1B Analysis of surface parametrizations for modern photometric stereo modeling [9534-36]

9534 1C Some illumination models for industrial applications of photometric stereo [9534-83]

9534 1D Photometric stereo sensor for robot-assisted industrial quality inspection of coated composite material surfaces [9534-16]

9534 IE MATLAB toolbox for the regularized surface reconstruction from gradients [9534-44]

9534 IF Perspective photometric stereo beyond Lambert [9534-52]

\section{WORKSHOP APPLICATIONS OF THERMOGRAPHY IN INSPECTION CONTEXT}

9534 IG 3D and NDT using scanning from heating [9534-66]

$9534 \mathrm{1H} \quad$ Outdoor thermal monitoring of large scale structures by infrared thermography integrated in an ICT based architecture [9534-95]

$953411 \quad$ A comparative study of in-situ measurement methods of a building wall thermal resistance using infrared thermography [9534-97]

$9534 \mathrm{lJ} \quad$ Temperature measurement by IR camera of heated device to high temperature during a short time [9534-99]

$95341 \mathrm{~K} \quad$ Glued structures inspection based on lock-in thermography [9534-101]

$95341 \mathrm{~L}$ Applications of the thermography in the animal production [9534-102] 


\section{Authors}

Numbers in the index correspond to the last two digits of the six-digit citation identifier (CID) article numbering system used in Proceedings of SPIE. The first four digits reflect the volume number. Base 36 numbering is employed for the last two digits and indicates the order of articles within the volume. Numbers start with 00, 01, 02, 03, 04, 05, 06, 07, 08, 09, 0A, 0B...0Z, followed by 10-1Z, 20-2Z, etc.

\author{
Aberra Guebrou, S., OD \\ Aigueperse, Antoine, $0 Z$ \\ Albouy-Kissi, Benjamin, OB \\ Aldea, Emanuel, 13 \\ Al-Habaibeh, Amin, 11 \\ Aoki, Yoshimitsu, 18 \\ Arciniegas, Andres F., 04 \\ Aruga, Haruki, OS \\ Aubreton, O., 02, 0U, $1 \mathrm{G}$ \\ Augereau, B., 07 \\ Autrique, Laurent, $1 \mathrm{~K}$ \\ Averty, Rodolphe, $1 \mathrm{H}$ \\ Babu Rao, C., 12 \\ Badshah, Amir, 0J \\ Barmaimon, Daniel, 19 \\ Barnabé, Pierre, 00 \\ Belkacemi, M., $1 G$ \\ Ben Said, Ahmed, ON \\ Bernard, A., OD \\ Bharti, Vibhav, 19 \\ Blanik, Nikolai, OP \\ Blazek, Vladimir, OP \\ Brancheriau, Loïc, 04 \\ BreuB, Michael, IF \\ Bringier, Benjamin, 08 \\ Bugarin, F., Ol \\ Candau, Yves, 11 \\ Cariou, Claude, 14 \\ Chaitanya, G. M.S. K., 12 \\ Chandrakumar, Chanjief, 11 \\ Chanussot, J., 03 \\ Chatillon, Sylvain, 10 \\ Chehdi, Kacem, 14 \\ Clark, Daniel, 19 \\ Colicchio, Bruno, OV \\ Coniglio, N., 02 \\ Cornille, N., Ol \\ Cortes, Yolima, 04 \\ Cremers, Daniel, 1B \\ Crinière, Antoine, $1 \mathrm{H}$ \\ Cudel, Christophe, OV \\ Dalla Mura, M., 03 \\ Daval, V., OU \\ Debayle, Johan, 09 \\ Deng, Zhiyuan, OV \\ Désage, Simon-Frédéric, 06 \\ Destrez, Raphaël, OB \\ Devy, Michel, $\mathrm{OH}$ \\ Dhali, Maruf Ahmed, 19
}

Díaz, Immaculada, $1 \mathrm{~L}$

Dislaire, Godefroid, 00

Drumetz, L., 03

Dubosclard, Pierre, $\mathrm{OH}$

Dumoulin, Jean, $1 \mathrm{H}$

Durou, Jean-Denis, $1 \mathrm{C}$

Duvieubourg, Luc, 16

Edard, Pierrick, 1J

Eitzinger, Christian, 1D

El Moubtahii, R., 07

Espinosa, Luis F., 04

Fasogbon, Peter, 16

Fauvet, Eric, OK

Favrelière, Hugues, 06

Fernandez-Maloigne, C., 05, 07

Fertil, Bernard, 09

Feuillet, Vincent, 11

Fofi, David, $\mathrm{OZ}$

Foufou, Sebti, ON, OT

Fougerolle, Yohan, $\mathrm{OZ}$

Freixenet, Jordi, OA

Frelin, Fabrice, 06

Fujino, Masahiro, 1A

Gain, P., OD

Garcia, Frederic, OR

Garcia, Rafael, 15

Gaudy-Marqueste, Caroline, 09

Gavet, Yann, OD, OE

Gilblas, Rémi, OY

Ginhac, Dominique, 17

Gomez, E., OG

Gonzalez-Castro, Victor, 09

Granda, Guillermo, OM

Grob, Jean-Jacques, 09

Gyohten, Keiji, OW

Hadjidj, Rachid, ON

Haeberlé, Olivier, OV

Hagen, Oksana, 19

Hannachi, Ammar, OX

Harker, Matthew, $1 \mathrm{E}$

Harouna S., Aboubacar, 08

Hashimoto, Manabu, OS

$\mathrm{He}, \mathrm{Z}$., OD

Herbulot, Ariane, $\mathrm{OH}$

Heyrman, Barthélémy, 17

Hirsch, Ernest, OX

Hittawe, Mohamad Mazen, OL

Houssineau, Jérémie, 19

Ibos, Laurent, 1 | 
loannidis, Dimosthenis, $O Q$

Ipanaque, William, OM

Istenič, Klemen, 19

Jayakumar, T., 12

Jovančević, Igor, OY

Judic, Jean-Pierre, $1 \mathrm{~J}$

Kamal, Khurram, 11

Kato, Kunihito, 1A

Khalfaoui, Souhaiel, $\mathrm{OZ}$

Khan, Muhammad A. U., OF

Khan, Taiq M., OF

Khanian, Maryam, IF

Khoudeir, Majdi, 08

Kimura, Tokihiro, OW

Kitamura, Yuka, OS

Kohler, Sophie, OV, OX

Kong, Yinan, OF

Konik, Hubert, $\mathrm{OH}$

Kudo, Yuta, 18

Kumar, Anish, 12

Lacassagne, Lionel, 10

Lacaze, Pierre-Antoine, 16

Laligant, Olivier, OK

Lallement, Alex, OX

Lambert, Jason, 10

Lapray, Pierre-Jean, 17

Larnier, Stanislas, $\mathrm{OH}$

Lauffenburger, Jean-Philippe, OV

Le Goïc, Gaëtan, 06

le Hégarat, Sylvie, 13

Lemaître, Guillaume, OA, OC

Leonhardt, Steffen, OP

Leroy, Sophie, 00

Likothanassis, Spiros, $0 Q$

Lombard, S., 03

LU, Qinglin, OK

Lucas, Yves, OB

Luengo, L., OG

Macaire, Ludovic, 16

Machacuay, Jorge, OM

Maire, Jean-Luc, 06

Manso, Alberto, $1 \mathrm{~L}$

Martí, Joan, $\mathrm{OC}$

Martí, Robert, OA

Martínez, R. A., 05

Marzani, Franck, 15

Massich, Joan, OA, OC

Mathavan, Senthan, 11

Mathieu, A., 02

Mecca, Roberto, 1B

Mériaudeau, Fabrice, OA, OC, OL

Meulenyzer, S., 03

Mirbach, Bruno, OR

Monchau, Jean-Pierre, 11

Montalvo, Gema, $1 \mathrm{~L}$

Morales, Joaquín, $1 \mathrm{~L}$

Morel, Olivier, 15

Mura, Emi, 1A

Nagai, Hajime, $1 \mathrm{~A}$

Naigeon, N., OD
Ohki, Hidehiro, OW

O'Leary, Paul, 1E

Omri, F., OT

Orteu, Jean-José, Ol, OY

Pataia, G., OD

Paul, Michael, OP

Perez, Laetitia, $1 \mathrm{~K}$

Pillet, Maurice, 06

Piñeiro, Carlos, $1 \mathrm{~L}$

Pinoli, Jean-Charles, OD, OE

Pirard, Eric, 00

Pitard, Gilles, 06

Prieto, Flavio A., 04, 0M

Purnachandra Rao, B., 12

Quéau, Yvain, 1C

Rahim, Mehdi, 09

Rahman, Mujib, 11

Rannou, Klervi, OE

Rastgoo, Mojdeh, 15

Richard, N., 05

Riou, Cécile, OV

Rodolà, Emanuele, 1B

Rogotis, Savvas, $0 Q$

Rougeron, Gilles, 10

Samper, Serge, 06

Sashida, Takehiko, 18

Sasi, B., 12

Schockaert, Cedric, OR

Sentenac, Thierry, OY

Seulin, Ralph, $\mathrm{OZ}$

Shahzad, Danish, OJ

Sharifi Boroujerdi, Ali, IF

Sidibé, Désiré, OA, OL

Sonneck-Museux, Nathanaëlle, IJ

Soto, Juan, OM

Stöger, Matthias, ID

Stolz, C., 02, $1 \mathrm{G}$

Sueda, Naomichi, OW

Taher, Akar, 14

Tairi, H., 07

Taniguchi, Rin-ichiro, OW

Thuret, G., OD

Treuillet, Sylvie, OB, OG

Truchetet, F., OU

Tzovaras, Dimitrios, $\mathrm{OQ}$

Ullah, Shan, 0J

Vaheesan, Kanapathippillai, 11

Vergé, Philippe, 1J

Viana, I., 0

Vilanova, Joan C., OA

Vizcaino, Elena, $1 \mathrm{~L}$

Walker, Paul M., OA

Wazaefi, Yanal, 09

Weigl, Eva, 1D

Zakharova, Anatasia, OK

Zambal, Sebastian, ID 


\title{
Conference Committee
}

\author{
Conference Chair
}

F. Meriaudeau, Université de Bourgogne (France)

Conference Co-chair

O. Aubreton, Université de Bourgogne (France)

International Scientific Committee

Ph. Bolon, General Chair, Université de Savoie (France)

K. Umeda, General Co-chair, Chuo University (Japan)

C. Stolz, Track Chair, Université de Bourgogne (France)

H. Muller, Track Chair, HSO-Valais (Switzerland)

K. Niel, Track Chair, University of Applied Sciences (Austria)

S. A. R. S. Abu Bakar, Universiti Teknologi Malaysia (Malaysia)

K. Aoki, Chukyo University (Japan)

Y. Aoki, Keio University (Japan)

T. Asano, Hiroshima Institute of Technology (Japan)

L. Autrique, Université d'Angers (France)

A. Baskurt, INSA Lyon (France)

P. R. Bingham, Oak Ridge National Laboratory (United States)

P. Bourgeat, CSIRO (Australia)

A. Chalifour, Université Trois Rivières (Canada)

L. d. F. Costa, Universidade São Paulo (Brazil)

C. Demonceaux, Université de Bourgogne (France)

J. Dubois, Université de Bourgogne (France)

J. D. Durou, Université Toulouse (France)

N. Escudeiro, Instituto Superior de Engenharia do Porto (Portugal)

D. Fofi, Université de Bourgogne (France)

J. Freixenet, Universitat de Girona (Spain)

D. Ginhac, Université de Bourgogne (France)

S. Gleason, Oak Ridge National Laboratory (United States)

I. Gurov, Saint Petersburg University (Russian Federation)

M. Hashimoto, Chukyo University (Japan)

Y. Hara, Nihon University (Japan)

A. Ishii, Kagawa University (Japan)

K. Kato, Gifu University (Japan)

S. Kaneko, Hokkaido University (Japan)

T. P. Karnowski, Oak Ridge National Laboratory (United States)

M. Khoudeir, Université de Poitiers (France)

O. Laligant, Université de Bourgogne (France)

S. Madenda, Gunadarma Universitas (Indonesia) 
X. Maldague, Université Laval (Canada)

R. Marti, Universitat de Girona (Spain)

H. Muller, HSO-Valais, (Switzerland)

J. Massich Vall, Université de Bourgogne (France)

V. Murino, Istituto Italiano Tecnologia (Italy)

K. Nidal, Universiti Teknologi Petronas (Malaysia)

A. Nakamura, Tokyo Denki University (Japan)

P. O'Leary, Institute for Automation (Austria)

V. Paquit, Oak Ridge National Laboratory (United States)

J.-C. Pinoli, École des Mines de Saint-Étienne (France)

E. Pirard, Université de Liège (Belgium)

E. Prasetyo, Gunadarma Universitas (Indonesia)

A. M. Saeed, Universiti Teknologi Petronas (Malaysia)

T. Shimizu, University of Yamanashi (Japan)

D. Sidibe, Université de Bourgogne (France)

S. Takahashi, Kagawa University (Japan)

H. Takimoto, Okayama Prefectural University (Japan)

K. Terada, Tokushima University (Japan)

S. Treuillet, Université Orléans (France)

K. Umeda, Chuo University (Japan)

Atsushi Yamashita, The University of Tokyo (Japan)

H. Zha, Beijing University (China)

Local Organizing Committee

O. Morel, Finance Chair, Université de Bourgogne (France)

R. Seulin, Industrial Chair, Le2i, CNRS, Université de Bourgogne (France)

D. Fofi, Publicity Chair, Université de Bourgogne (France)

D. Ginhac, Web Chair, Université de Bourgogne (France)

O. Aubreton, Workshop Chair, Université de Bourgogne (France)

N. Choffay, Secretary, Le2i, CNRS, Université de Bourgogne (France)

M. Belkacemi, Université de Bourgogne (France)

V. Daval, Université de Bourgogne (France)

C. Demonceaux, Université de Bourgogne (France)

E. Fauvet, Université de Bourgogne (France)

M. Hittawe, Université de Bourgogne (France)

O. Laligant, Université de Bourgogne (France)

L. F. C Lew Yan Voon, Université de Bourgogne (France)

G. Lemaitre, Université de Bourgogne (France)

Q. Lu, Université de Bourgogne (France)

J. Massich, Université de Bourgogne (France)

M. Rastgo, Université de Bourgogne (France)

C. Stolz, Université de Bourgogne (France)

D. Strubel, Université de Bourgogne (France)

S. Naffouti, Université de Bourgogne (France) 


\section{Introduction}

Researchers from the Le2i, Laboratory of Electronics, Computer Science and Computer Vision (France), Le Creusot, are proud to host the Twelfth International Conference on Quality Control by Artificial Vision in Le Creusot. Since its launch in 1993, where the first edition was held in Le Creusot, the conference has traveled worldwide. It has been organized in the following places: Le Creusot, France in 1995, 1997, 2001, 2007; Japan in 1998, 2005 and 2013; Canada in 1999; the United States in 2003; Austria 2009, and Saint Étienne, France in 2011. QCAV is now back for its twentieth International anniversary to the city where it was born.

This year, in parallel with the conference, two workshops were held. One dealt with photometric stereo (organized by J. R. Durou and Y. Quéau), and one related to the applications of thermography in inspection context (organized by L. Autrique and L. Pérez).

Thus as a whole, fifty-six papers were selected from among seventy-eight submissions by the International Committee (seven of which were from the poster session) from: Austria, Australia, Qatar, France, Japan, Pakistan, United Kingdom, Spain, Columbia, Germany, Greece, India, Luxembourg, Morocco, Peru, Belgium, Sri Lanka, Saudi Arabia, Mexico, and Italy.

This year, the conference was honored with the presence of three renowned guest speakers: Prof. François Goudail, Graduate School (France) Prof. E. Trucco, University of Dundee (Scotland) and Prof. J. Chanussot, GIPSA-Laboratory Grenoble, Institut Universitaire de France (France), who delievered a talk in each of our main tracks: Non Conventionnal Imaging Systems, Track Chair C. Stolz (France), Machine Vision, Track Chair K. Niel (Austria), and Computer Aided Diagnosis, H. Mueller (Switzerland).

It is also our pleasure to announce a special issue of the Journal of Electronic Imaging published by SPIE, with guest editors Assoc. Prof. A. S. Malik, Universiti Teknologi Petronas (Malaysia) and Prof. F. Meriaudeau, Le2i, Université de Bourgogne (France), dedicated to QCAV and featuring selections based on the best papers presented at the conference, among other submissions.

We also would like to thank our partners and sponsor for the support of this conference: Le Site du Creusot: IUT Le Creusot et Centre Universitaire Condorcet (France); Université de Bourgogne (France); CNRS (France); La Ville du Creusot (France); La communauté Urbaine Creusot-Montceau (France); and Conseil Régional de Bourgogne (France).

The QCAV 2015 Organizing Committee 
Proc. of SPIE Vol. $9534953401-12$

Downloaded From: https://www.spiedigitallibrary.org/conference-proceedings-of-spie on 26 Apr 2023 Terms of Use: https://www.spiedigitallibrary.org/terms-of-use 